\title{
Atomistics of self-diffusion in liquid metals
}

\author{
Lianwen Wang* \\ School of Physical Science and Technology, Lanzhou University, 730000 Lanzhou, P. R. China
}

\begin{abstract}
Self-diffusion in liquids is not well understood because of the lack of a clear microscopic knowledge on liquids. Here, on basis of an atomic model for liquids that we concluded from crystal melting, possible atomic details of self-diffusion in liquid metals are proposed. Accordingly, the coefficient of self-diffusion is derived for liquid elements, in reasonable agreement with experimental data.
\end{abstract}

\section{Introduction}

Despite intensive efforts devoted for understanding liquid metals, as signified by the series of international conferences on liquid and amorphous metals (LAM) over the past 60 years, we still have to face the fact that the liquid state is "the hardest to characterize" in comparison with the crystal and the gas states according to the presentation by Frans Spaepen given at the end of LAM-16. Such a situation is traced back through the pre-LAM ages in the early $20^{\text {th }}$ century to the days of van der Waals around 1873 [1-4].

As a consequence, a paragraph on liquids written in 1967 by Norman Nachtrieb [5] can be reused today, without any changes, which is cited in the following: "The transport properties of liquids, together with structural and thermodynamic information, provide the experimental basis for theories of the liquid state. Conversely, models of the liquid state must be judged according to the success with which they account for the kinetic as well as equilibrium properties of liquids. In principle, the self-diffusion of liquid metals should provide clues to the structures and interatomic potentials. There now exist data for the selfdiffusion of about 11 liquid metals, some of indifferent quality. A few studies are probably sufficiently reliable to merit serious theoretical attention at this time, but it will be obvious that improved experimental methods, applied over much wider ranges of temperature, are needed to provide better data." [5]

Here in this work, present knowledge on self-diffusion is briefly reviewed and examined in Section 2. In Section 3, a model for self-diffusion in liquid metals is developed, on basis of a microscopic liquid model that we developed in recent years, and compared with available experimental data. Finally, recent progresses on a possible crossover in selfdiffusion above the melting point are discussed in Section 4. On this basis, we are trying to show that the present liquid model does help in accounting for the self-diffusion of liquid metals and, judged according to this, this liquid model is in a promising direction approaching the microscopic knowledge of liquids.

\footnotetext{
*E-mail: 1wwang@1zu.edu.cn
} 


\section{Present knowledge on self-diffusion of liquid metals}

Available methods for the measurement of self-diffusion coefficient of liquid metals are briefly reviewed in [6] in which the data on 12 elements, mostly reported before 1970, were also summarized. These data were measured on the ground and according to [7] and [8] the first microgravity measurement was performed in the 1970s. As a result the data could inevitably have been contaminated by convective flow in liquids [7-10] and hence show large scattering, see Figure 3 of [9] with the data on $\mathrm{Sn}$ as an example. Advanced techniques applying in situ monitoring via X-ray or neutron radiography were developed recently, which are applicable only for interdiffusion of alloys [11-13].

In comparison, under microgravity environment [7-9], at least gravity-driven convective flow could be suppressed and, although diffusion measurements were still not fully free of disturbs [7,9], the data obtained by two independent research groups showed good agreement, see Figure 2 of [9] also with Sn as an example.

Besides measurements under microgravity, quasielastic neutron scattering (QENS) [1419] was argued to probe "the dynamics on atomic length scales and on a picosecond time scale, short enough to be undisturbed by the presence of convective flow." [18] "In order to derive the self-diffusion coefficient from QENS one has to assume that concepts developed in the framework of hydrodyanmics [20], i.e. in the limit of small wavenumber $q$ values, are valid also at $q$ values accessible by QENS" [18] and the self-diffusion coefficient was then related to the width of the QENS signal $[18,21]$. It is however noted that the analyses in [20] were carried out by assuming that the molecules in liquids interact through "a twobody, additive, central potential" (on page 16 of [20] above Equation 2.1.1).

On page 242 of [22] E. G. D. Cohen wrote: "All kinetic theories are, what could be called, effective two-particle theories. For, in all cases, the many particle dynamics of the fluid is approximated by an effective two-particle problem" [22]. Along this line, a significant advance in the understanding of liquid state diffusion was reported by $\mathrm{M}$. Dzugutov [23] that "the diffusion coefficient, reduced to a dimensionless from by scaling by the atomic collision frequency and the atomic diameter, is uniquely defined by the excess entropy (over the ideal gas [24]), a measure of the number of accessible configurations of the system" [23]. Modifications of intermolecular potentials [24] and calculating methods for the excess entropy $[25,26]$ seemed to lead to better applicability of this scaling law; however, indeed the issue of liquid state diffusion still remained unresolved but was just transferred to the calculation of excess entropy in terms of intermolecular potential.

From computer simulation results the influence of the type of intermolecular potential used on the final output of self-diffusion coefficient were more visible, see e.g. Figure 3 of [17].

Now, for deriving self-diffusion coefficient either from the kinetic theory or computer simulation, we have to face the fact that "establishing quantitative, predictive connections between the knowledge of the strong, complicated, and continual interactions that operate in condensed phases on the one hand, and the observable macroscopic properties on the other hand, remains a major challenge" [27].

Other theoretical approaches to self-diffusion in liquid metals simply overlooked interatomic potentials and started from e.g. the heat of vaporization [28], the Debye temperature [5] or the excess volume of a liquid over its crystal counterpart [29]. These models [5, 28, 29] represent the non-activated formalism for self-diffusion [5], see Figure 1(a), and lead the temperature $T$ dependences of the coefficient of self-diffusion $D$ in liquids to be $D \sim T^{2}$ [28] or $D \sim T$ [5]. On basis of the microgravity data, Mathiak et al [30] preferred a $T^{2}$ dependence of $D$. 
In comparison, there is also an activated formalism [5] suggested qualitatively by $\mathrm{J}$. Frenkel [31] and generally observed in measurements [6, 18, 19], see Figure 1(b), which results in $\ln D \sim 1 / T$, i.e.

$$
D=D_{0} \exp \left(-\frac{Q_{S D}}{k T}\right)
$$

where $D_{0}$ is a pre-exponential factor, $Q_{\mathrm{SD}}$ the activation energy of self-diffusion and $k$ the Boltzmann's constant. At present, both $D_{0}$ and $Q_{\mathrm{SD}}$ are obtained by fitting the measured or simulated self-diffusion data; theoretical predictions are not successful.

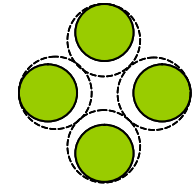

(a)

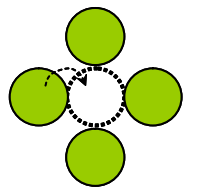

(b)

Fig. 1. Two-dimension sketchy diagram of atoms in a liquid with the free volume indicated by the dashed circle in (a). Although the non-activated formalism argued that the configuration of (a) might allow self-diffusion in liquids, we tend to advocate that self-diffusion in liquids should be an activated atomic jump process towards the nearby pre-formed vacancy (dotted circle in (b)), very alike to that in the corresponding crystal.

In Section 3, the coming and details of our microscopic model in favour of Equation (1) are introduced and, after this, $D_{0}$ and $Q_{\mathrm{SD}}$ are estimated and compared with available reported experimental data.

\section{Our microscopic model for self-diffusion in liquid metals}

\subsection{Starting point - estimating the energy sate of liquids}

In Section 2 it is seen that the major difficulty hindering the understanding of self-diffusion in liquids is the unsuccessfulness [1] in connecting classical interatomic potentials with the properties of liquids in which many-body potentials and many-body kinetics are present. We approached this issue by considering the continuity of liquids and corresponding crystals at the instant of melting [4] and proposed to estimate the energy state of liquids by using the energy of a vacancy divided among the first shell atoms [3]. By doing so, the many-body potentials are included in the formation energy of a vacancy, with which complex calculations were avoided to know liquid properties.

\subsection{Calculation of $D_{0}$}

Self-diffusion in liquid metals is supposed to consist of two steps, namely, formation of a vacancy and atomic jump to the vacancy. In our melting model, the vacancy formation energy was stored in the system at the instance of crystal melting signified by squashing of the vacancy $[32,33]$. On this basis we suggest that formation of a vacancy in liquids may need only the consumption of entropy but not the activation energy. After vacancy formation, the vacancy and its nearest neighbouring atoms return to a configuration similar to that before melting, see Figure 1(b), and for this reason the entropy consumption for 
vacancy formation in liquids is proposed to the entropy of fusion $S_{\mathrm{f}}$. Then the probability of finding a vacancy near an atom is:

$$
p_{\mathrm{V}}=\exp \left(-\frac{S_{\mathrm{f}}}{k}\right)
$$

The probability for an atomic jump to the pre-formed vacancy is suggested to be the same as that in a crystal:

$$
p_{\mathrm{m}}=\exp \left(\frac{S_{\mathrm{m}}}{k}-\frac{E_{\mathrm{m}}}{k T}\right)
$$

where $S_{\mathrm{m}}$ and $E_{\mathrm{m}}$ are the entropy and enthalpy of atomic migration. Then the time needed for an atomic jump in liquids is :

$$
t=\frac{1}{p_{\mathrm{V}} \cdot p_{\mathrm{m}} \cdot f_{0}}
$$

where $f_{0}$ is an attempting frequence normally in the order of $10^{12} \mathrm{~Hz}$. Finnally, with the Einstein's law for random walk $[34,35]$, the coefficient of self-diffusion is written as:

$$
D=\frac{d^{2}}{6 t}
$$

where $d$ is the atomic distance in the liquid.

By comparing Equations (1-5) one has:

$$
D_{0}=\frac{d^{2} f_{0}}{6} \exp \left(\frac{-S_{\mathrm{f}}+S_{\mathrm{m}}}{k}\right)
$$

In [36] (on page 3652) $S_{\mathrm{m}}$ was taken as $2.8 k, S_{\mathrm{f}}$ is taken from [6] and $d$ derived from the density of liquids reported in [6]; the obtained $D_{0}$ is compared with those reported in [6, 14, $16,19]$ in Figure 2. It is seen that the calculated and the reported $D_{0}$ are comparable in order of magnitude.

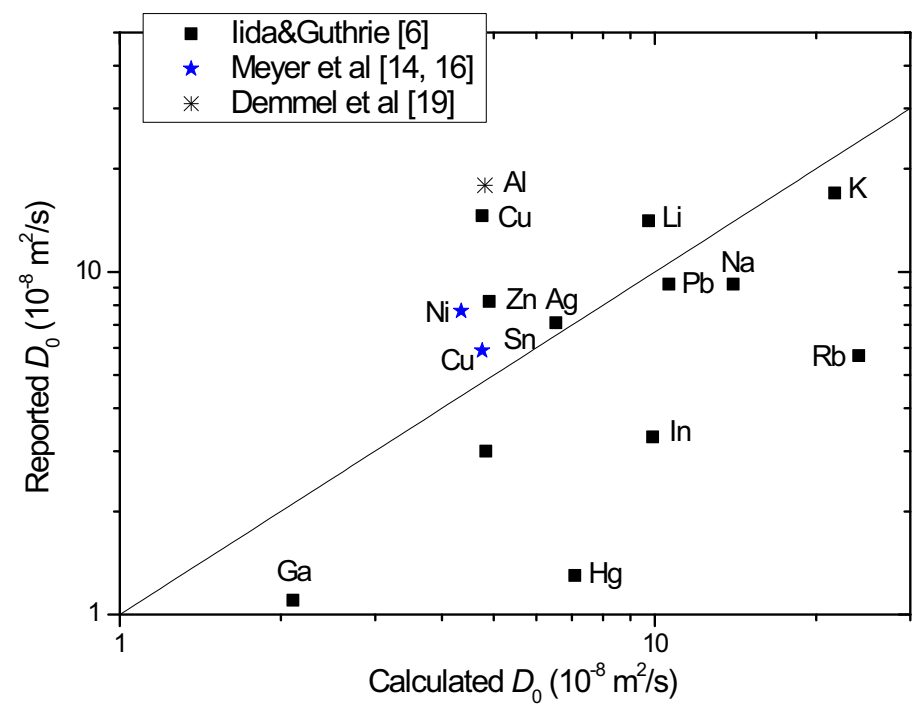

Fig. 2. Comparison of calculated $D_{0}$ by using Equation (6) with reported data. 


\subsection{Estimation of $Q_{\mathrm{SD}}$}

See Figure 3(left), at the instant of melting, a liquid nucleus was formed by the squashing of a vacancy by 2 of its $N$ nearest neighbouring migration atoms, with the energy of the activation energy for atomic migration $2 E_{\mathrm{m}}$ (approximately equals to the formation energy of a vacancy) stored in the system.

Self-diffusion in liquids may require that a vacancy be pre-formed, Figure 3(middle), with the consumption of the entropy of fusion. After this, the local atomic configuration returns to that before melting, but the energy $2 E_{\mathrm{m}}$ remains stored; if the liquid is at the melting point, now there are 2 atoms that can migrate to the vacancy.

Of course with temperature increasing the probability for an atom to migrate to the vacancy determined by Equation (3) will increase exponentially, however the present configuration may only allow 2 atoms to migrate because simultaneous migration of more than 2 atoms may squash the pre-formed vacancy which makes atomic migration impossible.

Consequently, self-diffusion in liquids should be a superdiffusion of 2 atoms, i.e. for each activation process 2 atoms can migrate. Accordingly the activation energy $Q_{\mathrm{SD}}$ for self-diffusion of liquid metals is estimated to be:

$$
Q_{\mathrm{SD}}=\frac{1}{2} E_{\mathrm{m}}
$$

By comparing the $E_{\mathrm{m}}$ data compiled in [37] and $Q_{\mathrm{SD}}$ reported in the literature, Equation (7) is in principle verified, see Figure 4. However, the data on Ge are left out for further considerations.
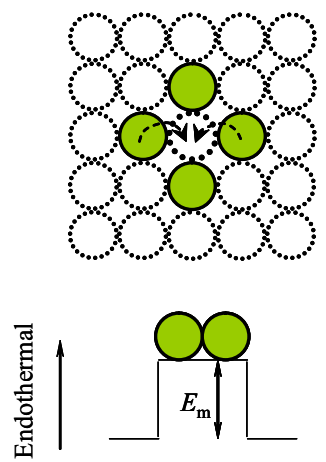

melting

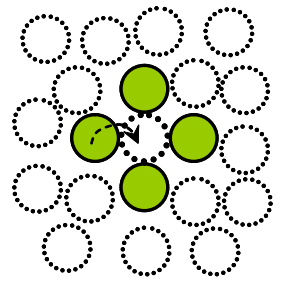

$Q_{\mathrm{SD}}=\frac{1}{2} E_{\mathrm{m}}$

$T_{\mathrm{m}}<T<T_{\mathrm{X}}$

self-diffusion in liquids

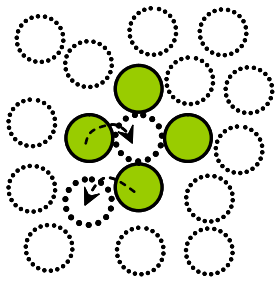

$Q_{\mathrm{SD}}=E_{\mathrm{m}}(?)$

$T>T_{X}$

Fig. 3. (left):Two-dimension sketchy diagram of atoms in a crystal (thin dotted circle) at the instant of melting and a liquid nucleus formed by squashing of a vacancy (thick dotted circle) when there are 2 out of the $N$ coordinating atoms of the vacancy are migratable.

(middle): For self-diffusion in liquids, after the pre-formation of the vacancy, for each activation process 2 atoms can migrate, and the activation energy of self-diffusion $Q_{\mathrm{SD}}$ may equal to $E_{\mathrm{m}} / 2$. (right) Only for discussion. When, with temperature increasing to above the crossover $T_{\mathrm{x}}$, two vacancies are available in the liquid nucleus, diffusion in liquids may turn to a simple activation behaviour, i.e. $Q_{\mathrm{SD}} \sim E_{\mathrm{m}}$. 


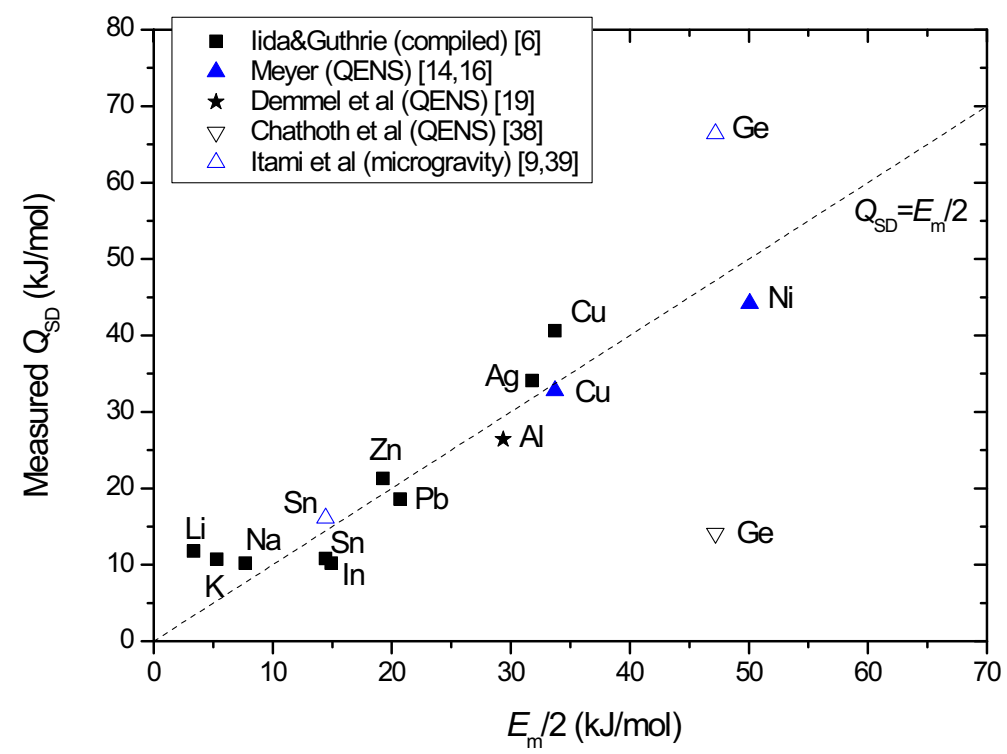

Fig. 4. Correlation between the reported activation energy for self-diffusion in liquid metals and the measured activation energy for atomic migration in crystals $E_{\mathrm{m}}[37]$

\section{Discussion}

When, with temperature increasing, two vacancies are available in the liquid nucleus, diffusion in liquids may require a simultaneous formation of two vacancies. Otherwise, e.g. if only one vacancy is formed, there is still excess entropy (over the crystal counterpart) left in the liquid nucleus so that the formed vacancy will soon be squashed and atomic diffusion just could not proceed. For such a situation, self-diffusion in liquids should then turn to normal self-diffusion, leading $Q_{\mathrm{SD}}$ to $E_{\mathrm{m}}$.

The scenario proposed above is very interesting in comparison with self-diffusion in crystals for which atomic jump is rare and is only possible for atoms in an activated energy state. Here, in liquids, it is seen that nearly all atoms have the energy to jump into a vacancy. However it seems that atomic migrations are too intensive so that if more than 2 atoms migrate simultaneously, the pre-formed vacancy may be squashed again. On the other hand, at high temperatures, if the excess entropy over the crystal is not completely squeezed out, a vacancy just could not be formed, which also does not permit self-diffusion.

In order to find out where the possible crossover temperature $T_{\mathrm{x}}$ may locate, an equation for the temperature dependence of the concentration of vacancies $C_{\mathrm{V}}$ in liquids is written as:

$$
C_{\mathrm{V}}=\exp \left(\frac{S_{\mathrm{V}}}{k}+\frac{S_{\mathrm{f}}}{k}-\frac{E_{\mathrm{V}}}{k T}\right)
$$

where $S_{\mathrm{V}}$ and $E_{\mathrm{V}}$ is the entropy and enthalpy of vacancy formation. Here the entropy of fusion is added because after a vacancy is formed it will be squashed instantly by nearby migration atoms, like the process of melting. In [32] $S_{\mathrm{V}}$ is taken as $4.1 \mathrm{k}$ at the homogeneous melting point, together with $S_{\mathrm{f}}$ data from [6] and $E_{\mathrm{V}}$ data from [37], the concentration of vacancies in liquids could be calculated.

Since during melting a vacancy is squashed with its energy and volume stored in the liquids, the crossover temperature dividing Figure 3 (middle) and Figure 3(right) may be set as:

$$
C_{\mathrm{V}}\left(T_{\mathrm{x}}\right)=1 / N
$$


where $N$ is the number of first coordination atoms in the corresponding crystal. By using Equations (8) and (9) the crossover temperature $T_{\mathrm{x}}$ is calculated and plotted against the melting point $T_{\mathrm{m}}$ in Figure 5. A rough correlation between $T_{\mathrm{x}}$ and $T_{\mathrm{m}}$ is noticed namely $T_{\mathrm{x}} \sim 1.5 T_{\mathrm{m}}$.

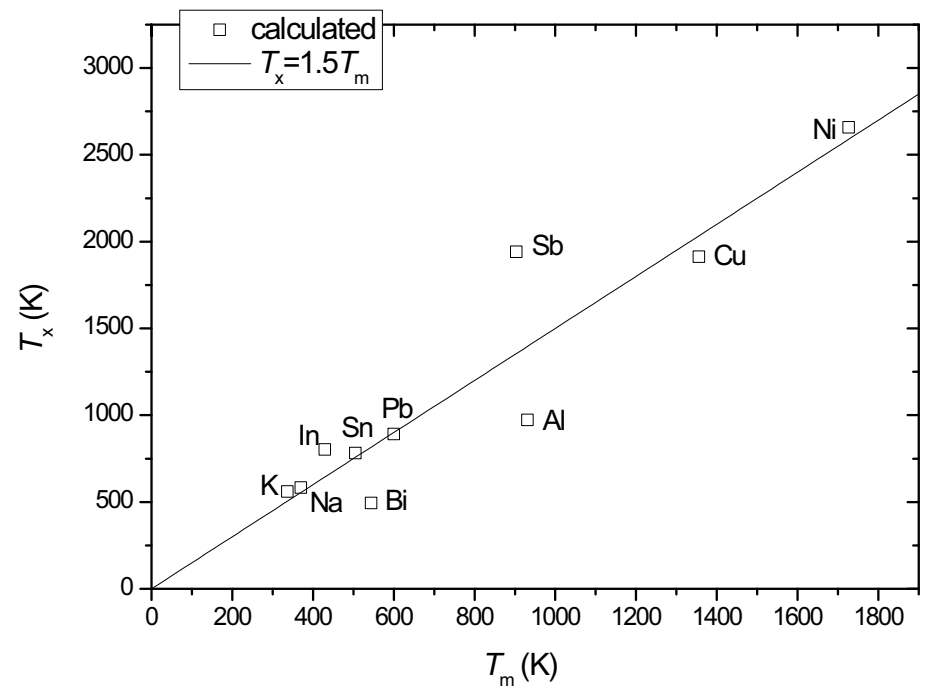

Fig. 5. Estimated crossover temperature $T_{\mathrm{x}}$ for self-diffusion in liquid metals.

Cautions are nevertheless needed for the present pure theoretical analyses and the tentative indications. Additional measurements in a wide temperature range are necessary when possible for further examination the temperature dependence of the coefficient of self-diffusion and the present analyses in comparison with others.

Anyway, in the present stage, two groups of research results are to be mentioned that may be related with the theoretical analyses carried out in this work. One is the neutron scattering evidences by Demmel et al for a dynamical crossover in liquid $\mathrm{Rb}$ [40], $\mathrm{Pb}$ [41], and $\mathrm{Al}[42]$ at around $1.5 T_{\mathrm{m}}$ which happens to coincide Figure 5 of this paper.

The other group of research results to be mentioned are the computer simulation outputs of Egami et al [43-45] for the dynamics of glass-forming liquids in which a crossover was also evidenced at about $1.5 T_{\mathrm{m}}[45]$.

Finally, recent $a b$ initio molecular dynamic simulations of 216 atoms showed that $Q_{\mathrm{SD}}$ for liquid $\mathrm{Ga}$ increased from 8 to $16 \mathrm{~kJ} / \mathrm{mol}$ at a temperature above the melting point [46].

It is less likely that the above results obtained over the years and the present theoretical analyses are not connected anyhow since all are focusing on one issue - a possible crossover in the kinetics of liquids above the melting point.

\section{Conclusion}

On basis of a simple microscopic model for crystal melting and for liquids, self-diffusion in liquid metals is analyzed to be an activated superdiffusion process via vacancies. The accordingly calculated prefactor and activation energy of self-diffusion are in general agreement with available experimental data. Furthermore, a possible crossover in the selfdiffusion of liquid metals above the melting point is discussed in terms of the present model. 
This work is supported by the Fundamental Research Funds for the Central Universities (grant number lzujbky-2015-120) and the National Natural Science Foundation of China (grant number 51101077).

\section{References}

1. J. L. Finney, Philos. Mag. 93, 3940 (2013)

2. L. W. Wang, AIP Conf. Proc. (LAM-15) 1673, 020013 (2015)

3. L. W. Wang, Metals 4, 570 (2014)

4. L. W. Wang, J. Non-Cryst. Solids 383, 59 (2014)

5. N. H. Nachtrieb, Adv. Phys. 16, 309 (1967)

6. T. Iida, R. I. L. Guthrie, The physical properties of liquid metals (Oxford University Press, New York, 1993)

7. G. Mathiak, E. Plescher, R. Willnecker, Meas. Sci. Technol. 16, 336 (2005)

8. T. Masaki, T. Fukazawa, S. Matsumoto, T. Itami, S. Yoda, Meas. Sci. Technol. 16, 327 (2005)

9. T. Itami, T. Masaki, H. Aoki, S. Munejiri, M. Uchida, S. Matsumoto, K. Kamiyama, K. Hoshino, J. Non-Cryst. Solids 312-314, 177 (2002)

10. L.B. Jalbert, R.M. Banish, F. Rosenberger, Phys. Rev. E 57, 1727 (1998)

11. B. Zhang, A. Griesche, A. Meyer, Phys. Rev. Lett. 104, 035902 (2010)

12. F. Kargl, M. Balter, Ch. Stenzel, Th. Gruhl, N. Daneke, A. Meyer, J. Phys.: Conf. Ser. 327, 012011 (2011)

13. F. Kargl, M. Engelhardt, F. Yang, H. Weis, P. Schmakat, B. Schillinger, A. Griesche, A. Meyer, J. Phys.: Condens. Matter 23, 254201 (2011)

14. A. Meyer, S. Stüber, D. Holland-Moritz, O. Heinen, T. Unruh, Phys. Rev. B 77, $092201(2008)$

15. J. Horbach, R. E. Rozas, T. Unruh, A. Meyer, Phys. Rev. B 80, 212203 (2009)

16. A. Meyer, Phys. Rev. B 81, 012102 (2010)

17. F. Kargl, H. Weis, T. Unruh, A. Meyer, J. Phys.: Conf. Ser. 340, 012077 (2012)

18. A. Meyer, EPJ Web Conf. 83, 01002 (2015)

19. F. Demmel, D. Szubrin, W.-C. Pilgrim, C. Morkel, Phys. Rev. B 84, 014307 (2011)

20. J. P. Boon, S. Yip, Molecular hydrodynamics (Dover Publications, New York, 1991)

21. A. G. Novikov, M. N. Ivanovskii, V. V. Savostin, A. L. Shimkevich, O. V. Sobolev, M. V. Zaezjev, J. Phys.: Condens. Matter 8, 3525 (1996)

22. E. G. D. Cohen, Physica A 194, 229 (1993)

23. M. Dzugutov, Nature 381, 137 (1996)

24. J. J. Hoyt, M. Asta, B. Sadigh, Phys. Rev. Lett. 85, 594 (2000)

25. A. Samanta, Sk. M. Ali, S. K. Ghosh, Phys. Rev. Lett. 92, 145901 (2004)

26. N. Jakse, A. Pasturel, Sci. Rep. 6, 20689 (2016)

27. M. Scott Shell, P. G. Debenedetti, F. H. Stillinger, J. Phys. Chem. B 108, 6772 (2004)

28. R. A. Swalin, Acta Metall. 7, 736 (1959)

29. M. H. Cohen, D. Turnbull, J. Chem. Phys. 31, 1164 (1959)

30. G. Mathiak, A. Griesche, K.H. Kraatz, and G. Frohberg, J. Non-Cryst. Solids 205-207, 412 (1996)

31. J. Frenkel, Trans. Faraday Soc. 33, 58 (1937)

32. L. W. Wang, L. Zhang, K. Lu, Philos. Mag. Lett. 85, 213 (2005)

33. L. W. Wang, Q. Wang, K. Q. Lu, Phlios. Mag. Lett. 87, 19 (2007)

34. A. Einstein, Ann. Phys. 17, 549 (1905)

35. P. Atkins, J. de Paula, Physical chemicstry, $8^{\text {th }}$ ed. (W. H. Freeman and Company, New York, 2006), Chapter 21.12

36. L. W. Wang, Philos. Mag. 93, 3648 (2013) 
37. H. J. Wollenberger, Point defects in R. W. Cahn, P. Haasen eds., Physical Metallurgy, 4th ed (Elsevier, Amsterdam, 1996)

38. S. M. Chathoth, B. Damaschke, T. Unruh, K. Samwer, Appl. Phys. Lett. 94, 221906 (2009)

39. T. Itami, H. Aoki, N. Higashimoto, T. Onogi, K. Sugimura, M. Kaneko, Y. Abe, Y. Arai, A. Tanji, M. Uchida, K. Iribe, H. Ando, K. Goto, N. Tateiwa, M. Koyama, T. Morita, H. Kawasaki, S. Yoda, N. Koshikawa, T. Masaki, H. Oda, T. Suzuki, T. Nakamura, Y. Nakamura, Int. J. Microgravity Sci. Appl. 16, 79 (1999)

40. F. Demmel, A. Diepold, H. Aschauer, C. Morkel, Phys. Rev. B 73, 104207 (2006)

41. F. Demmel, W. S. Howells, C. Morkel, J. Phys.: Condens. Matter 20, 205106 (2008)

42. F. Demmel, A. Fraile, D. Szubrin, W.-C. Pilgrim, C. Morkel, J. Phys.: Condens. Matter 27, $455102(2015)$

43. S.-P. Chen, T. Egami, V. Vitek, Phys. Rev. B 37, 2440 (1988)

44. T. Tomida, T. Egami, Phys. Rev. B 52, 3290 (1995)

45. A. Jaiswal, T. Egami, Y. Zhang, Phys. Rev. B 91, 134204 (2015)

46. L. H. Xiong, X. D. Wang, Q. Yu, H. Zhang, F. Zhang, Y. Sun, Q. P. Cao, H. L. Xie, T. Q. Xiao, D. X. Zhang, C. Z. Wang, K. M. Ho, Y. Ren, J. Z. Jiang, Acta Mater. 128, 304 (2017). 